\title{
Imaging Nanotechnology
}

David C. Bell ${ }^{1,2}$, Yue $\mathrm{Wu}^{3}$, Carl Barrelet ${ }^{3}$ and Charles M. Lieber ${ }^{3,4}$

${ }^{1}$ Center for Imaging and Mesoscale Structures; ${ }^{2}$ Department of Physics; ${ }^{3}$ Department of Chemistry and Chemical Biology; ${ }^{4}$ Division of Engineering and Applied Sciences

Harvard University, Cambridge, Massachusetts 02138, USA

Building devices atom by atom from the "bottom up" is a challenging process, but the imaging and examination of nanotechnology is equally as challenging a prospect. Nanoscale objects are by definition small, their physical properties being modified from the corresponding bulk material; similarly the imaging of these objects presents new difficulties. Considerations for imaging must be made based on the finite size of these objects and devices, and assumptions which are usually made when imaging a bulk sample must be discarded or at least greatly modified. Multislice calculations for simulating images of nano-clusters and supported particles have been reported but further efforts must be made for understanding the imaging from other nano-objects [1].

The imaging of silicon nanowires with HRTEM illustrates several unique problems; nanowires have been constructed by previously reported methods [2,3]. For very small wires with only a thickness of several nanometers (Fig. 1) conventional image interpretation based on the atomic structure image can, in certain situations be invalid. The finite size across the wire and corresponding thickness produces edge effects that indicate structural or crystallographic changes that may or may not be representative of the material. It is observed for very thin silicon nanowires that the FFT produced from the structure image shows forbidden reflections corresponding to the $1 / 3\{422\}$ peaks (Fig. 2). One possible explanation for these peaks is that because of the variations of unit cells along the cylindrical cross-section of the silicon nanowire these peaks are not eliminated as is the case for a bulk (assumed infinite) sample [3]. Such effects need to be considered and either confirmed or dismissed when interpreting the images from nanoscale objects. HRTEM image simulations of the nanowires can be based on modified multislice approach, which includes the effects of the reducing number of atoms towards the edge of the wire. The symmetry of the wire allows the calculation to employ a modified 2-dimensional shape function; our periodic object being modeled is now less periodic in two dimensions. The Fourier transform for a nanowire oriented along the $\mathrm{x}$ direction becomes;

$$
\mathrm{V}(x, y, z)=\mathrm{V}(\mathbf{y})=\boldsymbol{F} \boldsymbol{T}[\mathrm{V}(\mathrm{u})]
$$

where we have assumed that $z$ is the direction of the incident electrons, and $\mathrm{u}$ is the associated vector in Fourier space corresponding to the real space vector y, examples which will be presented. Imaging nanotechnology presents challenges; the interpretation of atomic structure images requires considerations of the physics involved in imaging and the effects of a 'finite' crystal size and hence not a completely periodic structure.

References

[1] G. Nihoul, H. Sack-Kongehl and J. Urban, Cryst. Res. Technol. 33 7-8 (1998) 1025.

[2] Y. Cui et al., Appl. Phys. Lett. 7815 (2001) 2214.

[3] L. J. Lauhon et al., Nature 420 (2002) 57. 

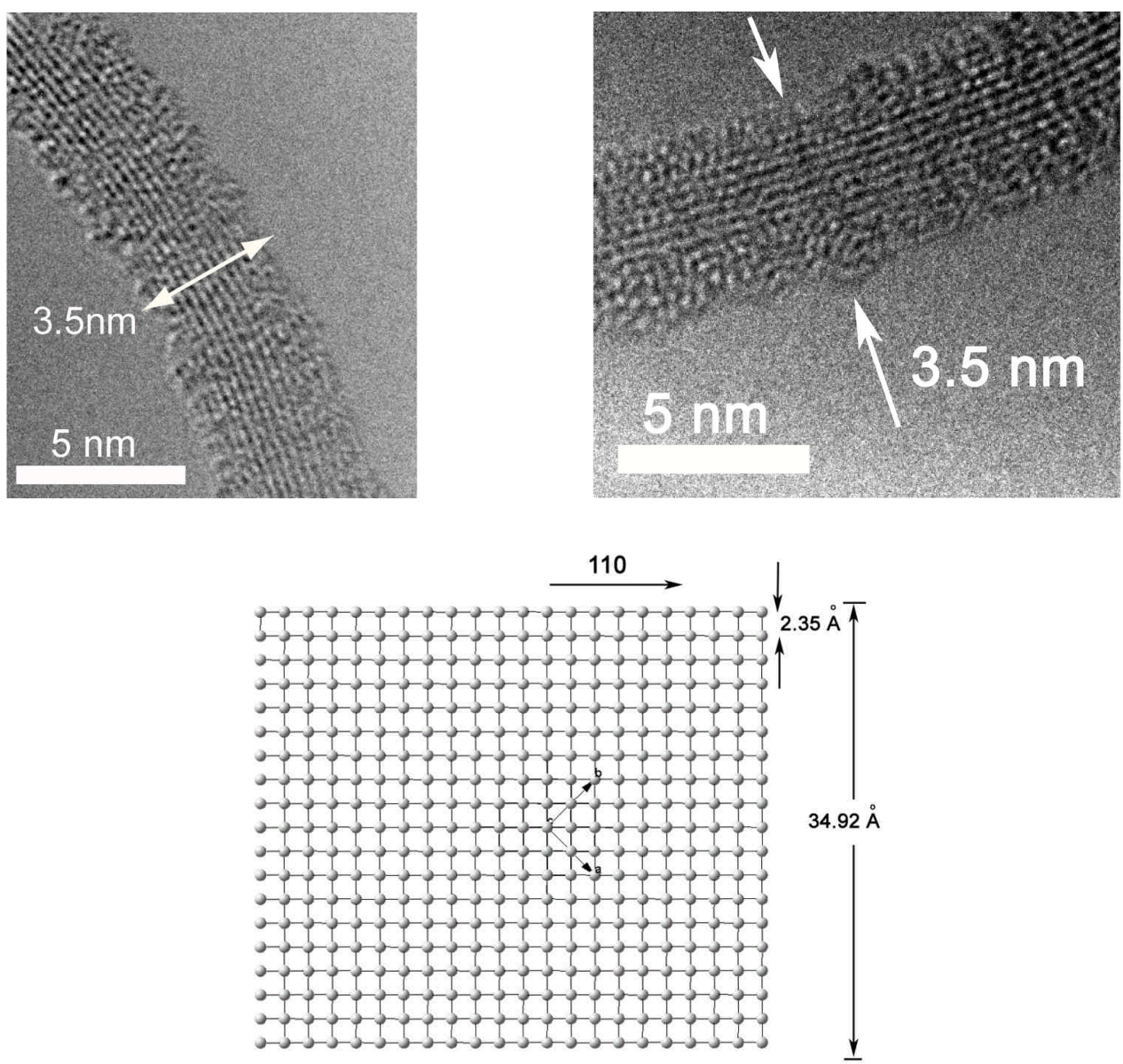

Fig. 1 (Top) HRTEM images of $3.5 \mathrm{~nm}$ silicon nanowires showing diffusion of contrast along the wire's edges. (Bottom) Diagram of a $3.5 \mathrm{~nm}$ nanowire along the [001] zone axis indicating that the maximum number of $\mathrm{Si}$ atoms is 19 for this projection which would occur through the center of the wire.
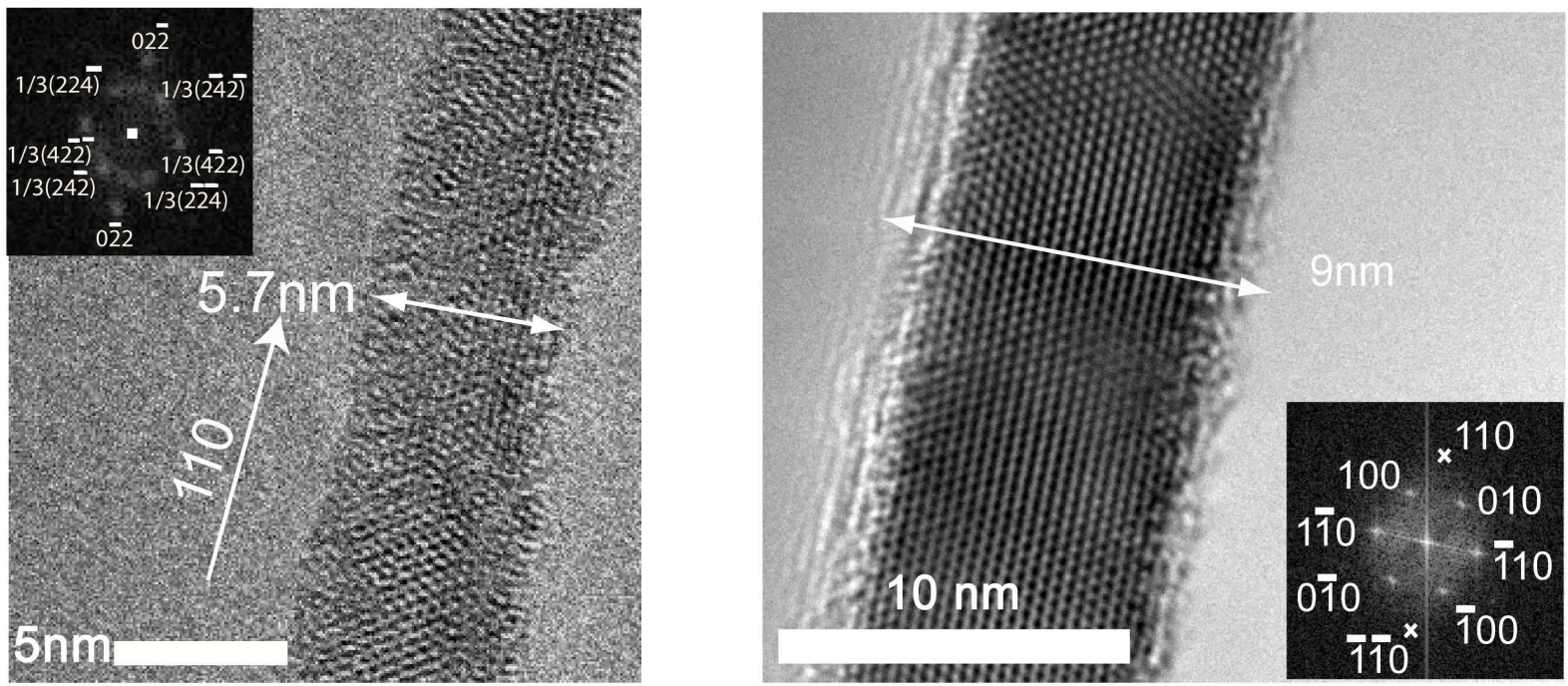

Fig. 2 (Left) HRTEM image of $5.7 \mathrm{~nm}$ silicon nanowire with corresponding FFT with forbidden reflections as indicated. (Right) Image of $9 \mathrm{~nm} \mathrm{CdS}$ nanowire with corresponding FFT. 